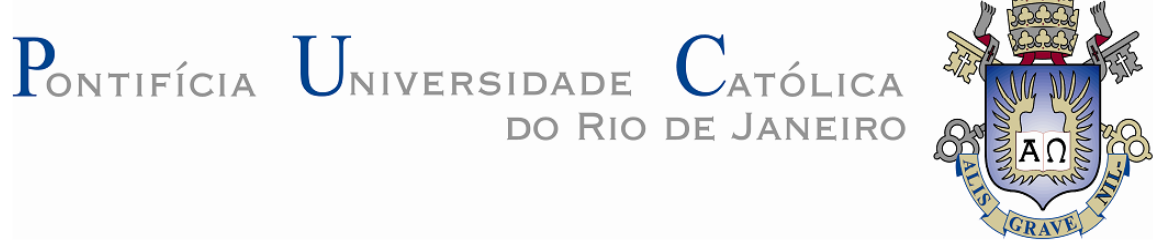

Marina Polónia Rios

\title{
Efeito de amortecedores no comportamento dinâmico de edifícios altos sob cargas de vento
}

Dissertação de Mestrado

Dissertação apresentada como requisito parcial para obtenção do grau de Mestre pelo Programa de PósGraduação em Engenharia Civil do Departamento de Engenharia Civil da PUC-Rio.

Orientador: Prof. Sebastião Artur Lopes de Andrade Co-orientador: Prof. Paulo Batista Gonçalves

Rio de Janeiro

Maio de 2015 


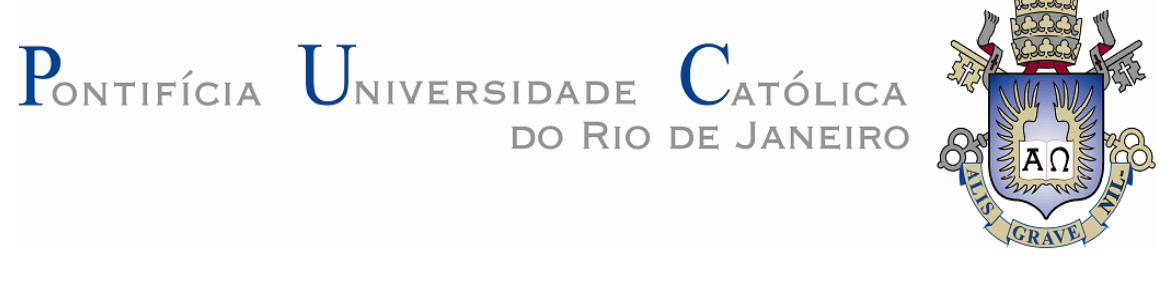

Marina Polónia Rios

\title{
Efeito de amortecedores no comportamento dinâmico de edifícios altos sob cargas de vento
}

Dissertação apresentada como requisito parcial para obtenção do grau de Mestre pelo Programa de Pós-Graduação em Engenharia Civil do Departamento de Engenharia Civil do Centro Técnico Científico da PUC-Rio. Aprovada pela Comissão Examinadora abaixo assinada.

\author{
Prof. Sebastião Artur Lopes de Andrade \\ Orientador \\ Departamento de Engenharia Civil - PUC-Rio \\ Prof. Paulo Batista Gonçalves \\ Co-orientador \\ Departamento de Engenharia Civil - PUC-Rio
}

Prof. Raul Rosas e Silva

Departamento de Engenharia Civil - PUC-Rio

Prof. José Guilherme Santos da Silva Universidade do Estado do Rio de Janeiro

Prof. José Eugenio Leal Coordenador Setorial do Centro

Técnico Científico - PUC-Rio

Rio de Janeiro, 8 de maio de 2015. 
Todos os direitos reservados. É proibida a reprodução total ou parcial do trabalho sem autorização da universidade, da autora e do orientador.

Marina Polónia Rios

Formou-se em Engenharia Civil na Pontifícia Universidade Católica do Rio de Janeiro (PUC-Rio) em 2011. Apresentou trabalho de pesquisa de análise da utilização de amortecedores como forma de reduzir o efeito do vento em edifícios altos.

Ficha Catalográfica

Rios, Marina Polónia

Efeito de amortecedores no comportamento dinâmico de edifícios altos submetidos a cargas de vento / Marina Polónia Rios ; orientador: Sebastião Artur Lopes de Andrade ; co-orientador: Paulo Batista Gonçalves. - 2015. 136 p.. : il. (color.) ; $30 \mathrm{~cm}$

Dissertação (mestrado)-Pontifícia Universidade Católica do Rio de Janeiro, Departamento de Engenharia Civil, 2015. Inclui bibliografia

1. Engenharia civil - Teses. 2. Estrutura. 3. Edifícios altos. 4. Dinâmica. 5. Vento. 6. Amortecedores. I. Andrade, Sebastião Artur Lopes de. II. Gonçalves, Paulo Batista. III. Pontifícia Universidade Católica do Rio de Janeiro. Departamento de Engenharia Civil. IV. Título.

CDD: 624 


\section{Agradecimentos}

Aos meus orientadores Prof. Paulo Batista Gonçalves e Prof. Sebastião Artur Lopes de Andrade, agradeço pela dedicação e estímulo durante a realização desse trabalho.

Aos meus colegas e amigos da Cerne Engenharia e Projetos, pela compreensão e apoio ao longo do período do mestrado. Agradeço em especial ao amigo Rafael Medeiros, por sua grande ajuda e incentivo na realização desse trabalho.

Agradeço aos meus pais e à minha irmã, por compreenderem e estarem sempre ao meu lado, acreditando na minha capacidade e dando-me força para conquistar o meu objetivo.

Ao meu namorado, Raoni, por seu carinho e dedicação ao longo desse período, me apoiando em todos os momentos.

Às minhas amigas, pelo incentivo para concluir esse trabalho.

A todos os professores, funcionários e colegas de mestrado, que, de alguma forma, contribuíram para a realização deste trabalho.

À PUC-Rio, pela concessão de Bolsa de Isenção de taxas escolares para a realização do curso. 


\section{Resumo}

Rios, Marina Polónia; Andrade, Sebastião Artur Lopes (Orientador); Gonçalves, Paulo Batista (Co-Orientador). Efeito de amortecedores no comportamento dinâmico de edifícios altos sob cargas de vento. Rio de Janeiro, 2015. 136 p. Dissertação de Mestrado - Departamento de Engenharia Civil, Pontifícia Universidade Católica do Rio de Janeiro.

O aumento da altura dos edifícios, aliado ao surgimento de materiais mais resistentes, faz com que as estruturas sejam cada vez mais esbeltas. Com isso, a ação do vento se torna um importante fator a ser considerado nesses projetos. A sua característica dinâmica provoca efeitos de vibração nas estruturas que devem ser analisados, em especial em relação ao conforto do usuário, afetado por deslocamentos e acelerações elevadas. Este estudo aborda a utilização de amortecedores fluidos como forma de reduzir a resposta dinâmica das estruturas submetidas a cargas de vento. A carga de vento consiste em um evento aleatório, devendo ser analisada estatisticamente. Desta forma, foi adotado o Método dos Ventos Sintéticos para definir o carregamento de vento aplicado à estrutura. Os amortecedores empregados na estrutura são fluidos, altamente viscosos, portanto seu comportamento pode ser considerado linear. A avaliação do comportamento da estrutura foi realizada pelo programa computacional Robot Structural Analysis. Foi feita uma análise estática afim de realizar o pré-dimensionamento da estrutura. Em seguida, fez-se uma análise dinâmica para a estrutura submetida ao carregamento de vento, com o objetivo de se analisar a influência dos amortecedores. Foram definidos cinco modelos estruturais, com diferentes configurações de amortecedores, de forma a encontrar a sua melhor distribuição na estrutura para reduzir a resposta a níveis aceitáveis de conforto para os usuários.

\section{Palavras-chave}

Edifícios altos; dinâmica de estruturas; cargas de vento; amortecedores fluidos. 


\section{Abstract}

Rios, Marina Polónia; Andrade, Sebastião Artur Lopes (Advisor); Gonçalves, Paulo Batista (Co-Advisor). Effect of Dampers on the Dynamic Behaviour of Tall Buildings under Wind Loads. Rio de Janeiro, 2015. 136 p.. MSc. Dissertation - Departamento de Engenharia Civil, Pontifícia Universidade Católica do Rio de Janeiro.

With the increase in building height and the development of more resistant materials, structures are becoming more flexible. This has made the consideration of wind loads an important factor to be considered in their projects. The dynamic characteristic of these loads causes important vibration effects in these structures due to their low vibration frequencies, which must be considered in design, especially regarding the users comfort, affected by high displacements and acceleration. This study analyses the use of fluid dampers in order to reduce the dynamic response of the structure under wind loading. The wind load is a random phenomenon, and must be studied statistically. In the present work the Synthetic Wind Method has been adopted in order to generate the variation of the wind load in time. The dampers applied to the structure are fluid dampers, highly viscous, so its behavior can be considered linear. The computer software Robot Structural Analysis is used to study the structural behavior. An analysis considering the wind as an equivalent static load is adopted for the preliminary design. Then, a dynamic analysis is conducted, considering the structure under a time varying wind loading, to investigate the effect of the fluid dampers on the response. Five models are investigated, with different configurations for the dampers, in order to define the best configuration and obtain acceptable levels of displacements and acceleration.

\section{Keywords}

Tall buildings; structural dynamics; wind load; fluid dampers. 


\section{Sumário}

1. Introdução 22

1.1. Considerações Iniciais 22

1.2. Importância e objetivos do estudo 24

1.3. Revisão Bibliográfica 25

1.4. Escopo do trabalho 31

2. O Vento e seu Efeito nas Estruturas 32

2.1. Variação do vento conforme a altura 33

2.2. Movimento turbulento dos ventos 35

2.3. Análise estática para cargas de vento 36

2.4. Resposta dinâmica da estrutura 37

2.5. Análise dinâmica para cargas de vento 38

2.5.1. Velocidade média do vento 38

2.5.2. Parcela flutuante da velocidade do vento 40

2.5.3. Método dos ventos sintéticos 44

2.6. Definição das cargas atuantes na estrutura em estudo 47

2.6.1. Carregamento de vento para o espectro de potência de $\begin{array}{ll}\text { Davenport } & 48\end{array}$

2.7. Demais efeitos provocados pelo vento 51

2.7.1. Efeito de vórtices $\quad 51$

2.7.2. Efeito de martelamento 52

2.7.3. Efeito de golpe 53

2.7.4. Efeito de galope 53

2.8. Conforto dos usuários 53

3. Amortecedores 56

3.1. Introdução 56

3.2. Amortecimento inerente às estruturas 57

3.3. Amortecedores passivos, ativos, semi-ativos e híbridos 58 
3.4. Resposta das estruturas a amortecedores passivos 60

3.5. Tipos de Amortecedores Passivos 61

3.5.1. Amortecedores de massa sintonizados (AMS) 61

3.5.2. Amortecedores líquidos sintonizados (ALS) 62

3.5.3. Amortecedores de impacto 63

3.5.4. Amortecedores viscoelásticos 63

3.5.5. Amortecedores por fricção 64

3.5.6. Amortecedores metálicos 65

3.5.7. Amortecedores fluidos 66

4. Análise Numérica 72

4.1. Apresentação do modelo 72

4.2. Casos estudados 80

4.2.1. Modelo $1 \quad 82$

4.2.2. Modelo $2 \quad 83$

4.2.3. Modelo $3 \quad 84$

4.2.4. Modelo $4 \quad 85$

4.2.5. Modelo 5

4.2.5.1. Modelo $5.1 \quad 87$

4.3. Análise dinâmica pelo método dos ventos sintéticos 88

4.3.1. Modelo inicial: definição do espectro de potência a ser adotado 88

4.3.2. Modelo $1 \quad 90$

4.3.3. Modelo $2 \quad 94$

4.3.4. Modelo $3 \quad 96$

4.3.5. Modelo $4 \quad 100$

4.3.6. Modelo 5

4.3.7. Modelo $5.1 \quad 108$

4.3.8. Resumo dos resultados 109

4.4. Variação do período de carregamento 109

4.4.1. Carga atuando em 25 segundos 110

4.4.2. Carga atuando em 50 segundos 111

4.4.3. Carga atuando em 100 segundos 112

4.4.4. Carga atuando em 150 segundos 113

4.4.5. Carga atuando em 200 segundos 114 
4.5. Crescimento gradual do carregamento 115

4.6. Modelo com mola e amortecedor em série 117

$\begin{array}{ll}\text { 4.7. Variação do ângulo de fase } & 118\end{array}$

4.8. Edifício submetido a cargas de vento calculadas pelo espectro $\begin{array}{ll}\text { de Kaimal } & 120\end{array}$

4.9. Carregamento no caso da ressonância 122

4.10. Comportamento na vibração livre 125

4.11. Análise Linear x Não Linear 126

$\begin{array}{ll}\text { 4.12. Análise dos esforços } & 128\end{array}$

$\begin{array}{ll}\text { 5. Considerações finais } & 129\end{array}$

6. Referências Bibliográficas 132 


\section{Índice de Figuras}

Figura 1.1 - Edifícios acima de 400 metros existentes.

(Wikipedia, 2014) 22

Figura 1.2 - World Trade Center (Wikipédia, 2014) 23

Figura 1.3 - Petronas Towers (Mendes, 2014) 23

Figura 2.1 - Variação do vento conforme a altura (Mendis et al., 2007) 33

Figura 2.2 - Modos de vibração da estrutura (Mendis et al., 2007) 38

Figura 2.3 - Mapa de isopletas da velocidade básica do vento $V_{0}$ (NBR 6123, 1988) 39

Figura 2.4 - Função periódica (Blessmann, 1998). 40

Figura 2.5 - Comparação de diversos espectros de potência

(Beça, 2010) 42

Figura 2.6 - Coeficiente de arrasto (NBR 6123, 1988) 46

Figura 2.7 - Carga de vento segundo o espectro de Davenport para $\mathrm{z}=36,0 \mathrm{~m}$

Figura 2.8 - Carga de vento segundo o espectro de Davenport para $\mathrm{z}=72,0 \mathrm{~m}$

Figura 2.9 - Carga de vento segundo o espectro de Davenport para $\mathrm{z}=108,0 \mathrm{~m}$

Figura 2.10 - Carga de vento segundo o espectro de Davenport para $z=144,0 \mathrm{~m}$

Figura 2.11 - Carga de vento segundo o espectro de Davenport para $\mathrm{Z}=172,8 \mathrm{~m}$

Figura 2.12 - Carga de vento segundo o espectro de Kaimal para $\mathrm{z}=36,0 \mathrm{~m}$

Figura 2.13 - Carga de vento segundo o espectro de Kaimal para $\mathrm{z}=72,00 \mathrm{~m}$

Figura 2.14 - Carga de vento segundo o espectro de Davenport para $\mathrm{z}=108,00 \mathrm{~m}$

Figura 2.15 - Carga de vento segundo o espectro de Kaimal para $\mathrm{Z}=144,00 \mathrm{~m}$

Figura 2.16 - Carga de vento segundo o espectro de Kaimal para $\mathrm{Z}=172,80 \mathrm{~m}$

Figura 2.17 - Efeito de desprendimento de vórtices

(Mendis et al., 2007)

Figura 2.18 - Graus de conforto, Segundo Chang (Blessmann, 1998) 54

Figura 3.1 - Curva de ressonância (Taylor, 1999)

Figura 3.2 - Amortecedor de Impacto (Kareem et al., 1999) 
Figura 3.3 - Amortecedor fluido (Soong \& Dargush, 1997) 66

Figura 3.4 - Taylor Device (Soong \& Dargush, 1997) 67

Figura 3.5 - Amortecedor de fluido viscoso esquemático

(Soong, Dargush, 1997) 68

Figura 3.6 - Torre Mayor: disposição dos amortecedores da fachada (Post, 2003)

Figura 3.7 - Travamento diagonal com amortecedor (Taylor Devices, 2014)

Figura 4.1 - Vista geral da estrutura do edifício

Figura 4.2 - Vista superior da estrutura do edifício 73

Figura 4.3 - Pilares da fachada $\quad 74$

Figura 4.4 - Pilares do core $\quad 74$

Figura 4.5 - Planta esquemática das vigas em cada pavimento 75

Figura 4.6 - Travamentos no projeto original - vista superior $\quad 75$

Figura 4.7 - Travamentos na direção $x$-Vista Frontal 76

Figura 4.8 - Travamentos na direção $y$ - Vista Frontal 76

Figura 4.9 - Disposição dos amortecedores na fachada - Modelo $1 \quad 82$

Figura 4.10 - Substituição de travamentos rígidos em X por barras com amortecedores - Modelo $2 \quad 83$

Figura 4.11 - Disposição dos amortecedores na fachada - Modelo $3 \quad 84$

Figura 4.12 - Disposição dos amortecedores na fachada - Modelo $4 \quad 85$

Figura 4.13 - Disposição dos amortecedores na fachada - Modelo $5 \quad 86$

Figura 4.14 - Vigas com ligações por rótula substituídas por ligações resistentes a momento - Modelo 5.1

Figura 4.15 - Comparação do deslocamento sofrido pela estrutura em seu topo submetida aos esforços de vento definidos pelos espectros de potência de Davenport e Kaimal

Figura 4.16 - Comparação da velocidade apresentada no topo da estrutura submetida aos esforços de vento definidos pelos espectros de potência de Davenport e Kaimal

Figura 4.17 - Comparação da aceleração apresentada no topo da estrutura submetida aos esforços de vento definidos pelos espectros de potência de Davenport e Kaimal

Figura 4.18 - Deslocamento no topo da estrutura do Modelo $1 \mathrm{com}$ amortecedores com $30 \% \mathrm{C}_{\mathrm{cr}}$

Figura 4.19 - Velocidade no topo da estrutura do Modelo $1 \mathrm{com}$ amortecedores com $30 \% \mathrm{C}_{\mathrm{cr}}$

Figura 4.20 - Aceleração no topo da estrutura do Modelo $1 \mathrm{com}$ amortecedores com $30 \% \mathrm{C}_{\mathrm{cr}}$

Figura 4.21 - Deslocamento no topo da estrutura do Modelo $2 \mathrm{com}$ amortecedores com $30 \% \mathrm{C}_{\mathrm{cr}}$

Figura 4.22 - Velocidade no topo da estrutura do Modelo $2 \mathrm{com}$ amortecedores com $30 \% \mathrm{C}_{\mathrm{cr}}$ 
Figura 4.23 - Aceleração no topo da estrutura do Modelo 2 com amortecedores com $30 \% \mathrm{C}_{\text {cr }}$

Figura 4.24 - Deslocamento no topo da estrutura do Modelo $3 \mathrm{com}$ amortecedores com $30 \% \mathrm{C}_{\mathrm{cr}}$

Figura 4.25 - Velocidade no topo da estrutura do Modelo $3 \mathrm{com}$ amortecedores com $30 \% \mathrm{C}_{\mathrm{cr}}$

Figura 4.26 - Aceleração no topo da estrutura do Modelo $3 \mathrm{com}$ amortecedores com $30 \% \mathrm{C}_{\mathrm{cr}}$

Figura 4.24 - Comparação dos deslocamentos das opções 2 e 3 com amortecedores com $30 \% \mathrm{C}_{\text {cr }}$

Figura 4.25 - Comparação das velocidades das opções 2 e 3 com amortecedores com $30 \% \mathrm{C}_{\mathrm{cr}}$

Figura 4.26 - Comparação das acelerações das opções 2 e 3 com amortecedores com $30 \% \mathrm{C}_{\mathrm{cr}}$

Figura 4.30 - Deslocamento no topo da estrutura do Modelo $4 \mathrm{com}$ amortecedores com $30 \% \mathrm{C}_{\mathrm{cr}}$

Figura 4.31 - Velocidade no topo da estrutura do Modelo $4 \mathrm{com}$ amortecedores com $30 \% \mathrm{C}_{\mathrm{cr}}$

Figura 4.32 - Aceleração no topo da estrutura do Modelo 4 com amortecedores com $30 \% \mathrm{C}_{\text {cr }}$

Figura 4.33 - Comparação dos deslocamentos das opções 3 e 4 com amortecedores com 30\%CcrFigura 4.34 - Comparação das velocidades das opções 3 e 4 com amortecedores com $30 \% \mathrm{C}_{\mathrm{cr}}$ Figura 4.35 - Comparação das acelerações das opções 3 e 4 com amortecedores com $30 \% \mathrm{C}_{\mathrm{cr}}$

Figura 4.36 - Deslocamento no topo da estrutura do Modelo $5 \mathrm{com}$ amortecedores com $30 \% \mathrm{C}_{\text {cr }}$

Figura 4.37 - Velocidade no topo da estrutura do Modelo 5 com amortecedores com $30 \% \mathrm{C}_{\mathrm{cr}}$

Figura 4.38 - Aceleração no topo da estrutura do Modelo $5 \mathrm{com}$ amortecedores com $30 \% \mathrm{C}_{\mathrm{cr}}$

Figura 4.39 - Comparação dos deslocamentos das opções 4 e 5 com amortecedores com $30 \% \mathrm{C}_{\mathrm{cr}}$

Figura 4.40 - Comparação das velocidades das opções 4 e 5 com amortecedores com $30 \% \mathrm{C}_{\mathrm{cr}}$

Figura 4.41 - Comparação das acelerações das opções 4 e 5 com amortecedores com $30 \% \mathrm{C}_{\mathrm{cr}}$

Figura 4.42 - Deslocamento no topo da estrutura do Modelo $5.1 \mathrm{com}$ amortecedores com $40 \% \mathrm{C}_{\mathrm{cr}}$

Figura 4.43 - Velocidade no topo da estrutura do Modelo $5.1 \mathrm{com}$ amortecedores com $40 \% \mathrm{C}_{\mathrm{cr}}$

Figura 4.44 - Aceleração no topo da estrutura do Modelo $5.1 \mathrm{com}$ amortecedores com $40 \% \mathrm{C}_{\mathrm{cr}}$ 
Figura 4.45 - Deslocamento no topo da estrutura do Modelo $5.1 \mathrm{com}$ amortecedores com $40 \%$ Ccr com a carga atuando por $25 \mathrm{~s}$

Figura 4.46 - Velocidade no topo da estrutura do Modelo $5.1 \mathrm{com}$ amortecedores com $40 \%$ Ccr com a carga atuando por $25 \mathrm{~s}$

Figura 4.47 - Aceleração no topo da estrutura do Modelo $5.1 \mathrm{com}$ amortecedores com $40 \% \mathrm{Ccr}$ com a carga atuando por $25 \mathrm{~s}$

Figura 4.48 - Deslocamento no topo da estrutura do Modelo $5.1 \mathrm{com}$ amortecedores com $40 \%$ Ccr com a carga atuando por $50 \mathrm{~s}$

Figura 4.49 - Velocidade no topo da estrutura do Modelo $5.1 \mathrm{com}$ amortecedores com $40 \%$ Ccr com a carga atuando por $50 \mathrm{~s}$

Figura 4.50 - Aceleração no topo da estrutura do Modelo $5.1 \mathrm{com}$ amortecedores com $40 \%$ Ccr com a carga atuando por $50 \mathrm{~s}$

Figura 4.51 - Deslocamento no topo da estrutura do Modelo $5.1 \mathrm{com}$ amortecedores com $40 \%$ Ccr com a carga atuando por $100 \mathrm{~s}$

Figura 4.52 - Velocidade no topo da estrutura do Modelo $5.1 \mathrm{com}$ amortecedores com $40 \%$ Ccr com a carga atuando por $100 \mathrm{~s}$

Figura 4.53 - Aceleração no topo da estrutura do Modelo $5.1 \mathrm{com}$ amortecedores com $40 \%$ Ccr com a carga atuando por $100 \mathrm{~s}$

Figura 4.54 - Deslocamento no topo da estrutura do Modelo $5.1 \mathrm{com}$ amortecedores com $40 \%$ Ccr com a carga atuando por $150 \mathrm{~s}$

Figura 4.55 - Velocidade no topo da estrutura do Modelo $5.1 \mathrm{com}$ amortecedores com $40 \%$ Ccr com a carga atuando por $150 \mathrm{~s}$

Figura 4.56 - Aceleração no topo da estrutura do Modelo $5.1 \mathrm{com}$ amortecedores com $40 \%$ Ccr com a carga atuando por $150 \mathrm{~s}$

Figura 4.57 - Deslocamento no topo da estrutura do Modelo $5.1 \mathrm{com}$ amortecedores com $40 \%$ Ccr com a carga atuando por $200 \mathrm{~s}$

Figura 4.58 - Velocidade no topo da estrutura do Modelo $5.1 \mathrm{com}$ amortecedores com $40 \%$ Ccr com a carga atuando por $200 \mathrm{~s}$

Figura 4.59 - Aceleração no topo da estrutura do Modelo 5.1 com amortecedores com $40 \%$ Ccr com a carga atuando por $200 \mathrm{~s}$

Figura 4.60 - Deslocamento no topo da estrutura do Modelo $5.1 \mathrm{com}$ amortecedores com $40 \% \mathrm{Ccr}$ com a carga com crescimento gradual

Figura 4.61 - Velocidade no topo da estrutura do Modelo $5.1 \mathrm{com}$ amortecedores com $40 \% \mathrm{Ccr}$ com a carga com crescimento gradual

Figura 4.62 - Aceleração no topo da estrutura do Modelo $5.1 \mathrm{com}$ amortecedores com $40 \% \mathrm{Ccr}$ com a carga com crescimento gradual Figura 4.63 - Deslocamento no topo da estrutura do Modelo 5.1 com $20 \%$ da rigidez original das diagonais e amortecedores com $40 \% \mathrm{Ccr}$

Figura 4.64 - Velocidade no topo da estrutura do Modelo $5.1 \mathrm{com}$ $20 \%$ da rigidez original das diagonais e amortecedores com $40 \%$ Ccr 118 Figura 4.65 - Aceleração no topo da estrutura do Modelo $5.1 \mathrm{com}$ $20 \%$ da rigidez original das diagonais e amortecedores com $40 \%$ Ccr 118 
Figura 4.66 - Comparação dos deslocamentos no topo da estrutura para carregamentos com diferentes ângulos de fases

Figura 4.67 - Comparação das velocidades no topo da estrutura para carregamentos com diferentes ângulos de fases

Figura 4.68 - Comparação das acelerações no topo da estrutura para carregamentos com diferentes ângulos de fases

Figura 4.69 - Deslocamento no topo da estrutura do Modelo 5.1 com amortecedores com $\mathrm{C}=40 \% \mathrm{C}_{\text {cr }}$ submetida a cargas de vento definidas pelo espectro de Kaimal

Figura 4.70 - Velocidade no topo da estrutura do Modelo $5.1 \mathrm{com}$ amortecedores com $\mathrm{C}=40 \% \mathrm{C}$ cr submetida a cargas de vento definidas pelo espectro de Kaimal

Figura 4.71 - Aceleração no topo da estrutura do Modelo $5.1 \mathrm{com}$ amortecedores com $\mathrm{C}=40 \% \mathrm{C}_{\text {cr }}$ submetida a cargas de vento definidas pelo espectro de Kaimal

Figura 4.72 - Comparação entre o deslocamento no topo da estrutura do Modelo 5.1 considerando o espectro de Davenport e de Kaimal 122 Figura 4.73 - Comparação entre a velocidade no topo da estrutura do Modelo 5.1 considerando o espectro de Davenport e de Kaimal

Figura 4.74 - Comparação entre a aceleração no topo da estrutura do Modelo 5.1 considerando o espectro de Davenport e de Kaimal Figura 4.75 - Deslocamento no topo da estrutura do Modelo 5 para o carregamento de ressonância

Figura 4.76 - Velocidade no topo da estrutura do Modelo 5 para o carregamento de ressonância

Figura 4.77 - Aceleração no topo da estrutura do Modelo 5 para o carregamento de ressonância

Figura 4.78 - Curva de ressonância dos deslocamentos para o Modelo 5

Figura 4.79 - Curva de ressonância das acelerações para a Modelo 5125

Figura 4.80 - Deslocamento no topo da estrutura do Modelo 5 para 0 carregamento de vibração livre

Figura 4.81 - Velocidade no topo da estrutura do Modelo 5 para o carregamento de vibração livre

Figura 4.82 - Aceleração no topo da estrutura do Modelo 5 para o carregamento de vibração livre

Figura 4.83 - Comparação dos deslocamentos para a análise linear e não linear

Figura 4.84 - Variação entre a diferença dos resultados para a análise linear e não linear 


\section{Índice de Tabelas}

Tabela 2.1 Coeficientes para as diversas características do terreno (Mendis et al., 2007)

Tabela 2.2 - Coeficientes $b, p$ e $F_{r, I l}($ NBR 6123, 1988)

Tabela 2.3 - Constantes para definição do carregamento de vento $\quad 47$

Tabela 2.4 - Percepção humana às vibrações (Medis et al., 2007) 55

Tabela 3.1 - Formas de reduzir a ação do vento (Kareem et al., 1999) 57

Tabela 3.2 - Amortecimento inerente às estruturas (NBR 6123, 1988) 58

Tabela 3.3 - Aplicações de amortecedores visco elásticos

(Kareem et al., 1999)

Tabela 3.4 - Aplicação de amortecedores por fricção (Kareem et al., 1999)

Tabela 3.5 - Estruturas com amortecedores metálicos (Kareem et al., 1999)

Tabela 3.6 - Edifícios com amortecedores para resistir ao vento (Taylor Devices, 2014)

Tabela 4.1 - Características dos mateirais

73

Tabela 4.2 - Modos de vibração natural da estrutura inicial 77

Tabela 4.3 - Resumo dos modelos estudados

Tabela 4.4 - Modos de vibração natural da estrutura com amortecedores - Modelo 1

Tabela 4.5 - Modos de vibração natural da estrutura com amortecedores - Modelo 2

Tabela 4.6 - Modos de vibração natural da estrutura com amortecedores - Modelo 3

Tabela 4.7 - Modos de vibração natural da estrutura com amortecedores - Modelo 4

Tabela 4.8 - Modos de vibração natural da estrutura com amortecedores - Modelo 5

Tabela 4.9 - Modos de vibração natural da estrutura com amortecedores - Modelo 5.1

Tabela 4.10 - Comparação da resposta no topo da estrutura submetida aos esforços de vento definidos pelos espectros de potência de Davenport e Kaimal

Tabela 4.11 - Resposta no topo da estrutura do Modelo $1 \mathrm{com}$ amortecedores com $10 \%$ C cr. $\mathrm{C}=7824 \mathrm{kNs} / \mathrm{m}$.

Tabela 4.12 - Resposta no topo da estrutura do Modelo $1 \mathrm{com}$ amortecedores com $20 \%$ C cr. C $=15648 \mathrm{kNs} / \mathrm{m}$. 
Tabela 4.13 - Resposta no topo da estrutura do Modelo $1 \mathrm{com}$ amortecedores com $30 \% \mathrm{C}_{\mathrm{cr}} \mathrm{C}=23472 \mathrm{kNs} / \mathrm{m}$.

Tabela 4.14 - Resposta da estrutura do Modelo 1 com barras sem amortecedores

Tabela 4.15 - Resposta no topo da estrutura do Modelo 2 com amortecedores com $10 \% \mathrm{C}_{\mathrm{cr}}$

Tabela 4.16 - Resposta no topo da estrutura do Modelo 2 com amortecedores com $20 \% \mathrm{C}_{\mathrm{cr}}$

Tabela 4.17 - Resposta no topo da estrutura do Modelo 2 com amortecedores com $30 \% \mathrm{C}_{c r}$

Tabela 4.18 - Resposta no topo da estrutura do Modelo $3 \mathrm{com}$ amortecedores com $10 \% \mathrm{C}_{\mathrm{cr}}$

Tabela 4.19 - Resposta no topo da estrutura do Modelo 3 com amortecedores com $20 \% \mathrm{C}_{c r}$

Tabela 4.20 - Resposta no topo da estrutura do Modelo 3 com amortecedores com $30 \% \mathrm{C}_{c r}$

Tabela 4.21 - Comparação das respostas das opções 2 e 3 com amortecedores com $30 \% \mathrm{C}_{\mathrm{cr}}$

Tabela 4.22 - Resposta no topo da estrutura do Modelo 3 com barras sem amortecedores

Tabela 4.23 - Resposta no topo da estrutura do Modelo 4 com amortecedores com $10 \% \mathrm{C}_{\mathrm{cr}}$

Tabela 4.24 - Resposta no topo da estrutura do Modelo 4 com amortecedores com $20 \% \mathrm{C}_{\text {cr }}$

Tabela 4.25 - Resposta no topo da estrutura do Modelo $4 \mathrm{com}$ amortecedores com $30 \% \mathrm{C}_{c r}$

100

Tabela 4.26 - Comparação das respostas das opções 3 e 4 com amortecedores com $30 \% \mathrm{C}_{\mathrm{cr}}$

102

Tabela 4.27 - Resposta no topo da estrutura do Modelo 4 com barras sem amortecedores

Tabela 4.28 - Resposta no topo da estrutura do Modelo 5 com amortecedores com $10 \% \mathrm{C}_{c r}$

Tabela 4.29 - Resposta no topo da estrutura do Modelo $5 \mathrm{com}$ amortecedores com $20 \% \mathrm{C}_{\mathrm{cr}}$

Tabela 4.30 - Resposta no topo da estrutura do Modelo 5 com amortecedores com $30 \% \mathrm{C}_{\mathrm{cr}}$

Tabela 4.31 - Comparação das respostas das opções 4 e 5 com amortecedores com $30 \% \mathrm{C}_{\mathrm{cr}}$

Tabela 4.32 - Resposta no topo da estrutura do Modelo $5 \mathrm{com}$ amortecedores com $40 \% \mathrm{C}_{\mathrm{cr}}$

Tabela 4.33 - Resposta no topo da estrutura do Modelo 5 com barras sem amortecedores

Tabela 4.34 - Resposta no topo da estrutura do Modelo $5.1 \mathrm{com}$ amortecedores com $40 \% \mathrm{C}_{c r}$ 
Tabela 4.35 - Resposta no topo da estrutura do Modelo $5.1 \mathrm{com}$ amortecedores com $40 \% \mathrm{C}_{\mathrm{cr}}$ e carga atuando por $25 \mathrm{~s}$

Tabela 4.36 - Resposta no topo da estrutura do Modelo $5.1 \mathrm{com}$ amortecedores com $40 \%$ Ccr e carga atuando por $25 \mathrm{~s}$

Tabela 4.37 - Resposta no topo da estrutura do Modelo $5.1 \mathrm{com}$ amortecedores com $40 \%$ Ccr e carga atuando por 50 s

Tabela 4.38 - Resposta no topo da estrutura do Modelo $5.1 \mathrm{com}$ amortecedores com $40 \%$ Ccr e carga atuando por $100 \mathrm{~s}$

Tabela 4.39 - Resposta no topo da estrutura do Modelo $5.1 \mathrm{com}$ amortecedores com $40 \%$ Ccr e carga atuando por 150 s

Tabela 4.40 - Resposta no topo da estrutura do Modelo $5.1 \mathrm{com}$ amortecedores com $40 \%$ Ccr e carga atuando por $200 \mathrm{~s}$

Tabela 4.41 - Resposta no topo da estrutura do Modelo $5.1 \mathrm{com}$ amortecedores com $40 \%$ Ccr e carga com crescimento gradual

Tabela 4.42 - Resposta no topo da estrutura do Modelo $5.1 \mathrm{com}$ $20 \%$ da rigidez original das diagonais e amortecedores com 40\% Ccr 117 Tabela 4.43 - Comparação dos deslocamentos no topo da estrutura para carregamentos com diferentes ângulos de fases

Tabela 4.44 - Comparação das velocidades no topo da estrutura para carregamentos com diferentes ângulos de fases

Tabela 4.45 - Comparação das acelerações no topo da estrutura para carregamentos com diferentes ângulos de fases

Tabela 4.46 - Resposta no topo da estrutura do Modelo $5.1 \mathrm{com}$ amortecedores com $\mathrm{C}=40 \% \mathrm{C}_{\text {cr }}$ submetida a cargas de vento definidas pelo espectro de Kaimal

Tabela 4.47 - Comparação entre a resposta no topo da estrutura do Modelo 5.1 com submetido a cargas de vento definidas pelo espectro de Davenport e de Kaimal

Tabela 4.48 - Resposta no topo da estrutura do Modelo 5 para o carregamento na ressonância

Tabela 4.49 - Redução dos esforços com amortecedores 


\section{Lista de Símbolos}

\section{Maiúsculas Romanas}

$A(\omega), B(\omega) \quad$ Componentes da transformada de Fourrier

C

Constante característica dos amortecedores

$C_{a} \quad$ Coeficiente de arrasto definido conforme NBR-6123

$C_{c r} \quad$ Coeficiente de amortecimento crítico da estrutura

$F_{r, I l} \quad$ Fator de rajada

L $\quad$ Dimensão característica

M Massa modal

$S_{1} \quad$ Fator topográfico

$S_{2}$

Fator que considera a influencia da rugosidade do terreno, das dimensões da edificação em estudo e de sua altura sobre o terreno

$S_{3} \quad$ Fator baseado em conceitos probabilísticos

$S_{t} \quad$ Número de Strouhal

$S^{v} \quad$ Espectro de potencia do vento

$T_{1} \quad$ Período natural principal da estrutura

V Velocidade de deslocamento do pistão

$\bar{V} \quad$ Velocidade média do vento

Vcr Velocidade crítica

$\bar{V}_{\mathrm{p}} \quad$ Velocidade de projeto

$\overline{V_{z}} \quad$ Velocidade do vento numa altura $Z$ acima da superfície

V0 Velocidade básica do vento

X1 Frequência adimensional

\section{Minúsculas Romanas}
$a_{0}, a_{n}, b_{n}$
Coeficientes da série de Fourrier
$B$
Parâmetro meteorológico usado na determinação de $S_{2}$ 

$b_{3}$ e $b_{600}$
Parâmetros meteorológicos para os períodos de 3 e $600 \mathrm{~s}$ respectivamente
$f \quad$ Frequência
$f_{1} \quad$ Frequência natural principal da estrutura
K Constante de Kármán
$P \quad$ Expoente da lei potencial da variação de $S_{2}$
$p^{\prime} \quad$ Pressão flutuante
$p_{3}$ e $p_{600} \quad$ Expoentes da lei potencial para os períodos de 3 e $600 \mathrm{~s}$ respectivamente
$\overline{\mathrm{q}}_{z} \quad$ Pressão dinâmica do vento, correspondente à velocidade característica $V_{k}$, em condições normais de pressão e de temperatura
$R \quad$ Número do harmônico ressonante com o primeiro modo de vibração da estrutura respectivamente
$z \quad$ Cota acima do terreno
$z_{0} \quad$ Coeficiente de rugosidade do terreno
$z_{g} \quad$ Altura da camada limite da atmosfera

\section{Minúsculas Gregas}
$\alpha$
Expoente característico do amortecedor
$\alpha$ e $\beta$
Constantes de integração de Newmark
Coeficiente de amortecimento natural da estrutura
$\theta$
Ângulo de fase aleatório
Comprimento de onda
$\mu$
Viscosidade do fluido
Amortecimento proporcional da estrutura
$\rho$
Densidade do fluido
$\sigma_{v}$
Desvio padrão da velocidade do vento
v
Velocidade axial do fluido 
Frequência natural de vibração do modo $\mathrm{n}(\mathrm{rad} / \mathrm{s})$

\section{Matrizes}

C

Matriz de amortecimento inerente da estrutura

K

Matriz de rigidez

$M$

Matriz de massa

$\Gamma$

Matriz de amortecimento passivo adicionado à estrutura

Vetores

$P$

Vetor de carregamento dinâmico do vento 


\section{Lista de Abreviaturas}

ABNT Associação Brasileira de Normas Técnicas

ALS Amortecedor de Líquido Sintonizado

AMS Amortecedor de Massa Sintonizado

ANSI American National Standards Institute

ASCE American Society of Civil Engineers

ASTM American Society for Testing and Materials

NBCC National Building Code of Canada 